\title{
Avaliação da fala pré-tratamento e pós-tratamento fonoaudiológico associado ao biofeedback ultrassonográfico de língua e de prótese bucomaxilofacial no câncer de cavidade oral
}

\author{
Evaluation of speech before and after speech therapy associated with
} ultrasound biofeedback of tongue and bucomaxillofacial prosthesis in

\author{
oral cavity cancer
}

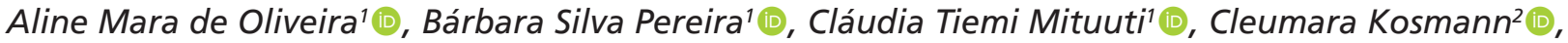 \\ Jaqueline Cardoso Estácio' ${ }^{10}$, Liliane Janete Grando ${ }^{3}$ (i)
}

\section{RESUMO}

As próteses bucomaxilofaciais (PBMF) têm como objetivo a reabilitação oral de pacientes com mutilações decorrentes de cirurgias de câncer de boca. Como parte dessa reabilitação oral, a terapia fonoaudiológica associada ao biofeedback ultrassonográfico da língua possibilita melhor precisão da produção de fala. O presente estudo teve por objetivo caracterizar a produção de fala de um indivíduo com histórico de múltiplas cirurgias oncológicas que levaram à glossectomia total, com auxílio de um dispositivo individualizado de acrílico, idealizado a partir da parceria Odonto-Fonoaudiologia da instituição e confeccionado por especialista em PBMF e terapia fonoaudiológica, utilizando o biofeedback visual com ultrassonografia. O indivíduo era homem, 45 anos, professor aposentado com histórico de carcinoma epidermóide de língua com episódios de recidiva. Como tratamento oncológico, foram realizadas diversas cirurgias associadas à radioterapia, ao longo de sete anos. O tratamento oncológico culminou com a glossectomia total e instalação de osteorradionecrose de mandíbula. $\mathrm{O}$ tratamento desta sequela da radioterapia também exigiu múltiplas abordagens cirúrgicas, com perda de grande parte da mandíbula, levando ao severo comprometimento das funções de deglutição e fala. Durante o atendimento multiprofissional da equipe Odonto-Fonoaudiologia de um hospital universitário, foi idealizada a confecção de um dispositivo individualizado de acrílico, objetivando melhorar a inteligibilidade da fala do paciente. Tal dispositivo foi confeccionado por dentista especialista em PBMF e ajustado em conjunto com a equipe de Fonoaudiologia. Com o dispositivo de acrílico adaptado, o indivíduo iniciou a terapia fonoaudiológica associada ao biofeedback, por meio da ultrassonografia de língua, com o objetivo de promover o refinamento da produção de fala dos fones fricativos [s] e []]. Ao comparar a avaliação pré-terapia e pós-terapia, foi possível identificar, após a análise das falas por juízes, melhora quanto à Porcentagem de Consoantes Corretas, de moderadamente severa para levemente moderada, bem como a inteligibilidade de fala, de insuficiente para regular. O dispositivo individualizado com a terapia fonoaudiológica associada ao biofeedback produziram resultados de fala satisfatórios, considerando-se a gravidade do caso e o elevado grau de mutilação do paciente.

Palavras-chave: Neoplasias de cabeça e pescoço; Glossectomia; Distúrbios da Fala; Ultrassonografia; Reabilitação

\begin{abstract}
The oral maxillofacial prostheses (PBMF) aim the oral rehabilitation of patients with mutilations resulting from oral cancer surgery. The oral rehabilitation was composed of speech therapy associated with ultrasound biofeedback of the tongue, which allows better precision in speech production. The present study aims to characterize the speech production of an individual with a history of multiple oncological surgeries with total glossectomy with the aid of an individualized acrylic device, designed based on the partnership between Dentistry and Speech Therapy and made by a prosthetic dentist, undergoing therapy and speech therapy with visual biofeedback by ultrasound. The individual is a 45 -year-old man, retired teacher with a history of tongue squamous cell carcinoma with episodes of recurrence. The cancer treatment was performed by several surgeries associated with radiotherapy over seven years. This treatment resulted in total glossectomy and the presence of osteoradionecrosis (ORN) of the mandible. The sequel to radiotherapy also required multiple surgical approaches with loss of a large part of the jaw, causing severe impairment of swallowing and speech functions. During the multidisciplinary care provided by the Dentistry and Speech Therapy team at a university hospital. The creation of an individualized acrylic device was conceived, aiming to improve the speech intelligibility of the patient. This device was made by a prosthetic dentist and adjusted together with the Speech Therapy team. With the adapted acrylic device, the individual started speech therapy associated with biofeedback by means of tongue ultrasound with the aim of promoting the refinement of the speech production of the fricative headphones [s] and [ $]$ ]. When comparing the pre-therapy and post-therapy assessment (after the analysis of the speeches by judges) it was possible to identify an improvement in the Percentage of Consonants Correct (PCC) from moderately-severe to slightly-moderate, as well as speech intelligibility from insufficient to regulate. The individualized device with speech therapy associated with biofeedback produced satisfactory speech results, considering the severity of the case and the high degree of mutilation of the patient.
\end{abstract}

Keywords: Head and Neck Neoplasms; Glossectomy; Speech Disorders; Ultrasonography; Rehabilitation

Estudo realizado na Universidade Federal de Santa Catarina - UFSC - Florianópolis (SC), Brasil.

${ }^{1}$ Departamento de Fonoaudiologia, Centro de Ciências da Saúde, Universidade Federal de Santa Catarina - UFSC - Florianópolis (SC), Brasil. ${ }^{2}$ Cirurgiã-dentista - Florianópolis (SC), Brasil.

${ }^{3}$ Departamento de Patologia, Universidade Federal de Santa Catarina - UFSC - Florianópolis (SC), Brasil.

Conflito de interesses: Não.

Contribuição dos autores: AMO e CTM contribuíram com a concepção do estudo, coleta e análise dos dados, escrita e revisão do manuscrito; BSP contribuiu com a coleta e análise dos dados e escrita do manuscrito; CK, JCE e LJG contribuíram com a análise da escrita e manuscrito e aprovação final da versão a ser publicada. Autor correspondente: Aline Mara de Oliveira. E-mail: aline.mara.oliveira@ufsc.br

Recebido: Junho 29, 2020; Aceito: Agosto 17, 2021 


\section{INTRODUÇÃO}

Epidemiologicamente, sabe-se que o câncer (CA) de boca atinge com maior frequência a população masculina a partir dos 40 anos de idade, devido a fatores de risco, como o tabagismo, o alcoolismo, o vírus do papiloma humano (HPV) e a predisposição genética. Os dados do Instituto Nacional do Câncer (INCA) ${ }^{(1)}$ no ano de 2018 apontam que o câncer de boca mais frequente é o carcinoma epidermoide, sendo que grande parte acomete a língua, variando em torno de $32 \%$ dos casos; os CAs de orofaringe com em torno de $18 \%$ de ocorrência e, por fim, o de assoalho de boca com cerca de $12 \%$.

$\mathrm{O}$ câncer de boca e seus tratamentos podem acarretar inúmeras sequelas indesejáveis e/ou irreversíveis no sistema estomatognático, dependendo do tipo do tratamento médico realizado. Entre essas consequências, destacam-se os defeitos cirúrgicos, a xerostomia, a fibrose dos tecidos, a necrose de tecido ósseo, o trismo e a disfonia, além de outros fatores que comprometem a qualidade de vida do indivíduo ${ }^{(2)}$.

No que diz respeito ao tratamento cirúrgico, a literatura demonstra que o maior volume de tecido ressecado na cavidade oral está associado ao melhor prognóstico de vida, mas também à piora na função de fala e de deglutição. As ressecções mandibulares segmentares têm um impacto significativo na eficiência da deglutição e podem afetar a produção da fala pela disrupção da integridade e da mobilidade dos articuladores. A ressecção segmentar da mandíbula geralmente ocorre em conjunto com ressecções extensas ou posteriores de língua, portanto, é provável que a piora da função esteja relacionada ao volume total de tecido ressecado e da porcentagem da base de língua ressecada ${ }^{(3)}$.

A radioterapia (RTx) adjuvante é indicada nos casos de cirurgias extensas de tumores com estadiamentos avançados em língua e base de língua, com comprometimento das estruturas que compõem o assoalho da boca e que necessitam de margens cirúrgicas de segurança que englobam parcialmente e/ou totalmente o arco mandibular, e que, dessa maneira, comprometem ainda mais as funções de fala e deglutição ${ }^{(4)}$. A depender do grau de comprometimento do paciente pela doença, pode-se, ainda, associar a quimioterapia (QTx). Ambos os tratamentos podem acarretar efeitos colaterais importantes, como a osteorradionecrose (ORN), também conhecida como necrose óssea avascular, necrose por radiação e/ou necrose isquêmica do osso, que, comumente, é associada às cirurgias extensas, como as de glossectomia e de mandibulectomia.

Esse tratamento adjuvante é indicado nos casos mencionados, já que, nesses casos, são necessárias margens cirúrgicas de segurança que englobam parcialmente e/ou totalmente o arco mandibular; do contrário, isso compromete ainda mais as funções de fala e deglutição ${ }^{(4)}$.

O impacto de tratamentos cirúrgicos associados à radioterapia e à quimioterapia para o tratamento do câncer de cabeça e pescoço (CCP) é evidente, principalmente em relação à fala. Portanto, compete ao fonoaudiólogo desenvolver estratégias de comunicação efetivas, como adaptação da fala ou de comunicação alternativa não verbal, com o intuito de proporcionar ao sujeito uma qualidade de vida relativamente melhor, promovendo a sua independência para atividades cotidianas e para a comunicação $0^{(5)}$.

Uma das opções de adaptação e de melhora da fala ocorre por meio do uso das próteses bucomaxilofaciais (PBMF), que permitem a reabilitação tanto estética, quanto funcional de pacientes com sequelas decorrentes de traumas, malformações congênitas ou pós-cirúrgicas de cânceres de cabeça e pescoço ${ }^{(6)}$. No caso de cirurgias que envolvem a língua, são descritas próteses pouco usuais, como as de língua ${ }^{(7)}$, e as mais usuais, como as próteses "rebaixadoras de palato".

Recentemente, o biofeedback por meio da ultrassonografia de língua foi inserido como ferramenta terapêutica nos casos de pacientes glossectomizados ${ }^{(8)}$. Nessa população, a maioria dos estudos inclui a ultrassonografia de língua associada à caracterização da produção de fala, envolvendo pacientes com $\mathrm{CCP}^{(9,10)}$. A ultrassonografia de língua fornece biofeedback cinemático, isto é, uma conexão entre o movimento da língua e o sinal auditivo correspondente. Sendo assim, o biofeedback proporciona um foco interno que controla a direção, o tempo e a força dos movimentos de língua, que produzem um determinado som de fala, e um foco externo que pode envolver a atenção do sujeito, a fim de resultar em um sinal acústico percebido pelo falante e seu ouvinte ${ }^{(11)}$.

Blyth et al. ${ }^{(8)}$ relataram que a imagem ultrassonográfica, durante as tarefas de fala, permite que os pacientes concentrem sua atenção no movimento da língua, aprendendo os ajustes necessários para melhorar a precisão da articulação. Os autores ainda ressaltaram que esse é um processo vantajoso, pois permite que o sujeito alcance a produção do som com mais elementos, aproximando-se, assim, da fala natural, a partir da estrutura resultante da glossectomia ${ }^{(8)}$.

Com base nos dados da literatura, observa-se escassez científica sobre a real efetividade do uso de PBMF em sujeitos tidos como "limite terapêutico" na reabilitação fonoaudiológica, assim como sobre a prática fonoaudiológica associada a novas tecnologias assistivas no processo de reabilitação com o uso da ultrassonografia de língua com biofeedback visual em pacientes glossectomizados. Considerando-se as características individuais de pacientes pós-cirúrgicos de câncer de cabeça e pescoço e as particularidades dos desfechos, há uma grande dificuldade no recrutamento de indivíduos com características semelhantes, que permitam a padronização de protocolos terapêuticos. Diante do exposto, ressalta-se que o presente estudo teve como objetivo caracterizar a produção de fala de um indivíduo com histórico de câncer de boca recidivado, tratado com múltiplas cirurgias e ciclos de RTx para tratamento do câncer e da osteorredianecrose, com o uso de um dispositivo confeccionado por especialista em PBMF. Além disso, propôs-se uma metodologia de intervenção utilizando o biofeedback visual com ultrassonografia.

\section{APRESENTAÇÃO DO CASO CLÍNICO}

Esta pesquisa foi aprovada pelo Comitê de Ética em Pesquisa com Seres Humanos (CEPSH), vinculado à Universidade Federal de Santa Catarina - UFSC, com a aprovação de número 3.686.654 e CAAE 63084016.8.0000.0121. O sujeito da pesquisa leu e assinou o Termo de Consentimento Livre e Esclarecido (TCLE).

O sujeito (A.) era do sexo masculino, com 45 anos de idade e professor aposentado. Em 2007, A. recebeu diagnóstico de neoplasia maligna de língua à esquerda, do tipo carcinoma epidermóide moderadamente diferenciado, e realizou a primeira intervenção cirúrgica por meio de hemiglossectomia à esquerda, com histórico de duas reconstruções microcirúrgicas: a primeira com retalho de músculo peitoral e a segunda, com retalho de músculo do braço. Todas as cirurgias foram realizadas pela equipe de cirurgia de cabeça e pescoço responsável pelo paciente. 
Nesse caso, foi indicada a RTx adjuvante, com 38 sessões e com dose total de radiação de 63 Gy pela técnica Intensity Modulated Radiation Therapy (IMRT) (ou Terapia de Radiação Modulada por Intensidade). Os resultados foram considerados satisfatórios, o paciente se alimentava por via oral e realizava acompanhamento multiprofissional no hospital oncológico de referência, porém, não foi possível obter informações precisas sobre o planejamento terapêutico durante esse período.

Em 2016, A. apresentou novo tumor, sendo submetido à mais uma intervenção cirúrgica de glossectomia parcial e mandibulectomia segmentar. Em 2018, após nova recidiva, ele foi submetido à glossectomia total e à nova mandibulectomia segmentar, remanescendo as regiões retromolar e de ramo ascendente da mandíbula (lado esquerdo) e região de pré-molares e molares inferiores (lado direito). Sendo assim, o paciente foi submetido a um novo protocolo de radiação por IMRT, com dose total de 60 Gy.

Após o tratamento oncológico da lesão primária recidivada, o paciente desenvolveu osteorradionecrose (ORN) na região do coto mandibular medial esquerdo. Episódios de agudização do quadro da ORN, associados ao menor aporte sanguíneo decorrente da RTx, causaram a ruptura da pele da face e a exteriorização do coto ósseo. Sendo assim, uma nova cirurgia, agora plástica reconstrutiva, foi realizada, com o intuito de controlar a ORN e de restabelecer a continuidade da pele da face.

$\mathrm{O}$ paciente se manteve em acompanhamento com equipe multidisciplinar, incluindo o fonoaudiólogo, durante todo o tratamento médico. As terapias fonoaudiológicas realizadas no serviço de origem possuíam como objetivo a reabilitação da fala e da deglutição, com o intuito de manter a alimentação por via oral, por meio de terapias indiretas e diretas da deglutição, uma vez que A. se alimentava por via alternativa (sonda nasoenteral). O paciente foi submetido ao exame objetivo da deglutição (videofluoroscopia), realizado devido à presença de complicações clínicas, como pneumonia aspirativa, e por causa da baixa evolução na reabilitação fonoaudiológica com terapia direta da deglutição. No exame de videofluoroscopia, foi possível verificar a presença de disfagia orofaríngea grave, sem possibilidade de reintrodução de alimentação por via oral segura, em razão da presença de aspiração silente de todas as consistências, ausência de reflexo de proteção de vias aéreas e/ou tosse efetiva. Por isso optou-se pela via alternativa de alimentação de longa permanência (gastrostomia).

Após sua estabilização, em dezembro de 2018, A. foi encaminhado ao Ambulatório de Suporte Odontológico a Pacientes com Câncer de Boca, do Núcleo de Odontologia Hospitalar/Hospital Universitário - UFSC, para nova abordagem de fase aguda de ORN de mandíbula e devido à exposição do coto mandibular remanescente. Foram realizadas dez sessões de ozonioterapia, intercaladas com sessões de fotobiomodulação (laser de baixa potência) na área de tecidos moles do entorno do coto mandibular exposto, associada à antibioticoterapia direcionada por antibiograma, havendo melhora do quadro clínico local e ausência de drenagem purulenta.

Durante o acompanhamento pela equipe do Núcleo de Odontologia Hospitalar/Hospital Universitário - UFSC, verificouse que A. se alimentava exclusivamente pela gastrostomia, apresentando acúmulo da pouca saliva produzida em cavidade oral e sialorreia, bem como presença eventual de pigarro e voz molhada após a deglutição de saliva. No que se refere à qualidade vocal, esta se apresentava rugosa, hipernasal e, em alguns momentos, molhada. Além disso, acrescenta-se que A. não mostrava interesse na tentativa de introduzir alimentação por via oral, já que o enfoque da sua queixa no período era estritamente relacionado à inteligibilidade de fala. Já no que se refere à fala, por causa das diversas intervenções cirúrgicas de reconstrução da lesão decorrente por ORN, A. apresentava alterações de inteligibilidade de fala devido à ausência de estruturas de contato para formulação dos fones, comunicandose, na grande maioria do tempo, de forma escrita.

O caso foi discutido em equipe multiprofissional e o sujeito foi encaminhado para a confecção de um dispositivo de fala em acrílico individualizado, realizado por cirurgião-dentista especialista em prótese bucomaxilofacial, cujo objetivo era permitir a fala, por meio do toque do lábio inferior no referido aparato, proporcionando a produção de alguns pontos articulatórios.

Durante o processo, na primeira etapa, o dispositivo individualizado foi confeccionado com material de moldagem flexível a partir de alginato, com o intuito de facilitar os ajustes necessários. No momento da instalação do dispositivo provisório, a equipe de fonoaudiologia não estava presente. A participação na produção do dispositivo deu-se por meio de ajustes periódicos (uma vez por semana) de seu tamanho e posicionamento, a partir dos achados nas reavaliações informais da fala e produções sonoras, uma vez que os locais de produção do dispositivo e de atendimento fonoaudiológico eram diferentes. Após o ajuste ser considerado satisfatório pelo paciente e pela equipe, na segunda etapa deu-se a acrilização do dispositivo em laboratório e, posteriormente, novos ajustes finos em boca, no que diz respeito à altura do dispositivo, em relação ao ponto de contato com o lábio inferior.

Devido à pequena abertura da boca, à ausência de apoio em consequência da presença de menos de um terço da mandíbula e à ausência de dentes adequados, não foi possível confeccionar uma prótese lingual ou rebaixadora de palato. Assim sendo, o dispositivo utilizado não se enquadra nos casos apresentados na literatura, inclusive, não se encontra classificado no livrobase da $\mathrm{PBMF}^{(12)}$. Em vista disso, o referido dispositivo foi descrito como dispositivo individualizado de acrílico e não como prótese verdadeira, porém, permite que o lábio inferior toque o superior, melhorando parcialmente a função de fala, mas isso não substitui as partes perdidas. Logo, trata-se de um dispositivo dentossuportado, como pode ser visto na Figura 1.

No retorno ao ambulatório, com o uso do dispositivo de acrílico auxiliando na produção fônica, observou-se que houve melhora da inteligibilidade da fala, com possibilidade de realização de pontos articulatórios, como os dos sons labiodentais [f] e [v]. Além disso, o sujeito mencionou estar muito satisfeito com o dispositivo, com boa adaptação, e relatou melhora na comunicação.

Após a confecção do dispositivo e a sua adaptação, iniciou-se o processo terapêutico de treino de fala com produção articulatória dos fones-alvo, porém, observou-se que o paciente mantinha a evolução estável, ou seja, a terapia tradicional mostrou limite terapêutico, demonstrando dificuldade na propriocepção para realizar ajustes espontâneos de fala.

Diante disso, o sujeito foi encaminhado para terapia fonoaudiológica associada ao biofeedback por meio da ultrassonografia de língua, cujo objetivo terapêutico foi [s] e [S] com o uso do referido dispositivo, tendo em vista que, devido à falta de estruturas e de funções intraorais, como a ausência de vedamento labial e de pressão intraoral, não foi possível que o sujeito realizasse a produção de fones plosivos. Sendo assim, optou-se por fones com os quais o sujeito apresentou mais 

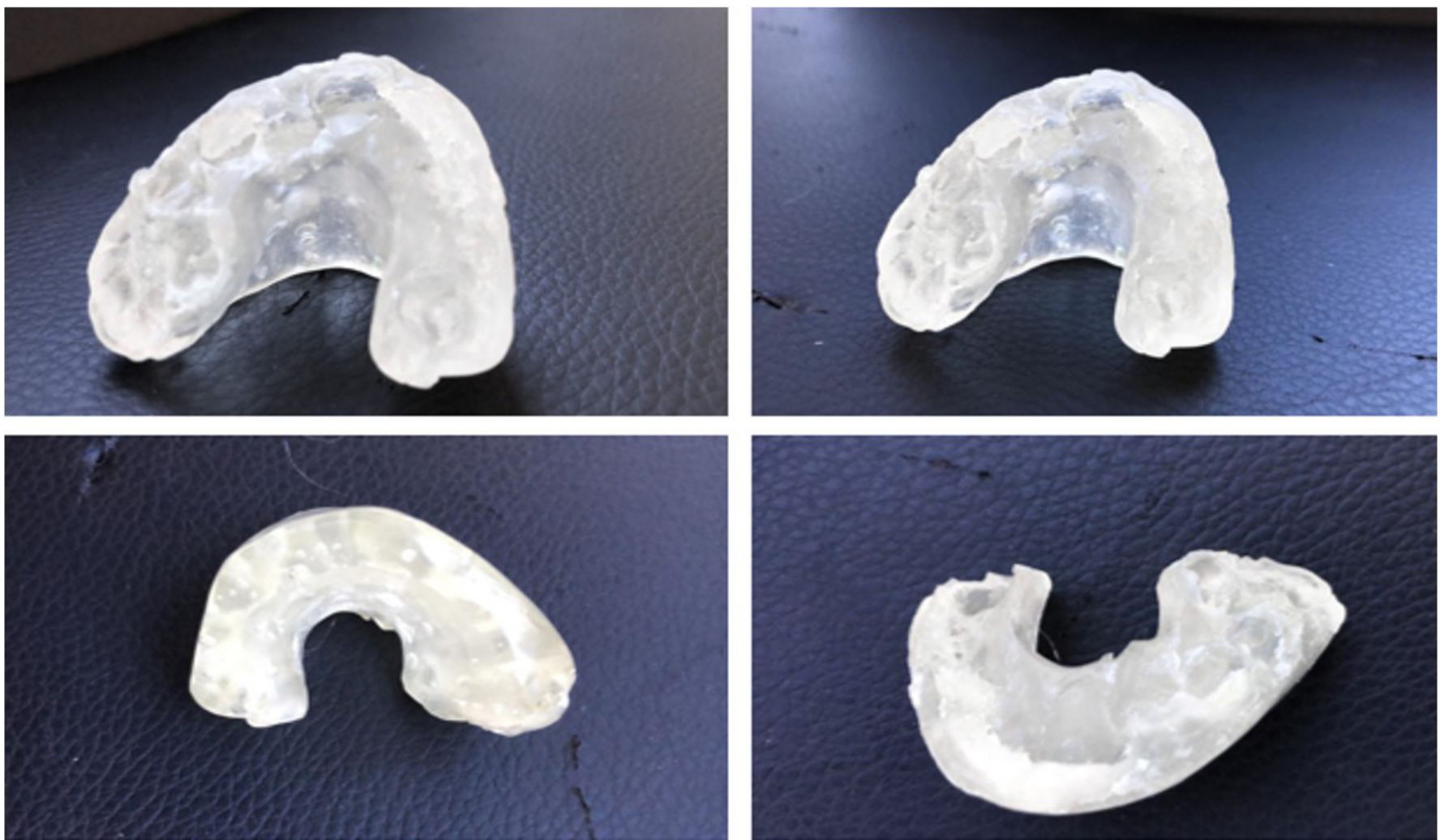

Figura 1. Dispositivo de fala em acrílico individualizado - prótese bucomaxilofacial

facilidade. As sessões de fonoterapia consistiam em demonstrar e caracterizar o contorno da neolíngua ao som-alvo isolado, primeiramente no terapeuta, com a intenção de que o paciente visualmente entendesse o segmento da produção do fone, e depois no paciente. Após esse processo, o paciente praticou a produção dos sons-alvo isolados e associados às vogais.

Além disso, a escolha pelos fones supracitados baseou-se em um estudo ${ }^{(8)} \mathrm{em}$ que os autores obtiveram resultados bem satisfatórios no que diz respeito ao treino de produção e de inteligibilidade desses fones. $\mathrm{O}$ estudo apresentou, ainda, a avaliação e o biofeedback por meio da ultrassonografia de língua, utilizando os seguintes equipamentos: microfone unidirecional; transdutor microconvexo acoplado a um aparelho de ultrassom portátil (Mindray M5); computador; sincronizador; caixa de som e estabilizador de cabeça (Articulate Instruments Ltd). O corpus utilizado para a avaliação perceptivo-auditiva foi: chave (/'Jave/), chica (/'Jika/), chuva (/' Juva/), sapo (/'sapo/), sica (/'sika/) e suco (/'suko/). Essas palavras foram representadas por figuras e apresentadas por meio do software Articulate Assistant Advanced (AAA) - (Articulate Instruments Ltd), a partir de diferença estatística indutiva e não descritiva. $\mathrm{O}$ sujeito foi instruído a incluir a palavra-alvo na frase-veículo "Digo bem bonito". Cada palavra-alvo foi repetida dez vezes no período pré-tratamento e dez vezes no período pós-tratamento, totalizando 120 palavras (seis palavras x 10 repetições x 2 sessões (avaliação e reavaliação) = 120 repetições).

$\mathrm{O}$ sujeito foi submetido à avaliação perceptivo-auditiva, por meio das palavras-alvo gravadas na avaliação ultrassonográfica, sendo que a gravidade das distorções das palavras foi baseada no cálculo do índice Porcentagem de Consoantes Corretas Revisado (PCC-R). A partir dos resultados obtidos no PCC-R, foi possível classificar a gravidade das distorções como: severa (PCC $<50 \%)$; moderadamente severa $(50 \%<$ PCC $<65 \%$ ), levemente moderada $(65 \%<\mathrm{PCC}<85 \%)$ e leve $(85 \%<$ PCC $<100 \%)^{(13)}$. A avaliação foi realizada por três juízes experientes em transcrição fonética, sem atuação em fononcologia. Os juízes atuam no ambulatório que analisa áudios e imagens de ultrassonografia de diversas alterações de fala e possuem experiência com análise articulatória. As amostras de fala foram disponibilizadas remotamente e a análise foi realizada separadamente, sem interação entre juízes, e em diferentes momentos.

Quanto ao grau de inteligibilidade de fala (GIF), foram utilizadas as seguintes marcações para cada palavra-alvo:

1. Insuficiente e/ou incompreensível, quando a maior parte da palavra é incompreendida, dificultando, assim, a compreensão da mensagem principal;

2. Regular e/ou pouco compreensível, quando os juízes compreendem metade da palavra e, desse modo, deduzem a mensagem principal;

3. Boa e/ou compreensível, quando é possível compreender toda a palavra de forma clara, bem como a mensagem principal $^{(14)}$.

Com os dados obtidos, realizou-se uma média para classificar a porcentagem dos resultados.

O sujeito foi submetido a dez sessões, compreendendo duas sessões para gravação da avaliação de fala e oito para a terapia propriamente dita. A terapia tinha duração de 50 minutos, sendo 30 minutos para o biofeedback visual a partir da ultrassonografia de língua e 20 minutos para a abordagem 
tradicional e treinamento para percepção auditiva da fala. A abordagem tradicional consistiu em complementar a abordagem do biofeedback ultrassonográfico e, para tal, utilizou-se a estratégia de bombardeio auditivo, adaptado para o sujeito, com os fones [s] e [J].

As palavras selecionadas para a estimulação dos segmentos [s] e [J], na abordagem tradicional, foram: sair, sala, salada, sapato, seco, celular, seguro, senha, seta, sério, sétimo, sinal, cinco, cinema e chove, choque, brechó, choro, chocolate, choupana, chuteira e machucou.

Para ambas as abordagens da terapia, baseadas no estudo de Blyth et al. ${ }^{(8)}$, foram utilizados níveis de complexidade linguística: de fones para sílabas, para palavras e, por fim, para sentenças envolvendo o som-alvo. O material de estímulo para a prática na terapia convencional foi obtido a partir de recursos on-line, realizados com palavras e textos com os fones-alvo, analisados cuidadosamente, verificando-se e respeitando-se os níveis de complexidade de articulação no momento da escolha.

A primeira sessão, sendo pré-terapia, constituiu-se na gravação ultrassonográfica dos movimentos da neolíngua na produção de frases balanceadas, contendo os fones brasileiros com o dispositivo. Da segunda até a sétima sessão, foram estimulados os fones [s] e [S], utilizando-se os critérios explicitados anteriormente. Da oitava à nona sessão, foram realizados os treinos de sentença para verificar a aplicação dos fones-alvo estimulados para analisar o nível de retenção dos fones trabalhados anteriormente. A décima sessão foi utilizada para a coleta da amostra de fala por meio da ultrassonografia, a fim de comparar a evolução e/ou aquisição do fone-alvo pelo sujeito (Quadro 1).

Com o apoio do biofeedback, a sessão da terapia se caracterizou em realçar o contorno da neolíngua relativo ao som-alvo isolado, ou seja, o terapeuta forneceu o modelo para o sujeito acerca da produção a ser atingida. Primeiramente, o terapeuta realizava o modelo de produção de fala; em seguida, higienizava a sonda e solicitava a realização de fala do paciente. Logo após o modelo, o sujeito o reproduziu durante as terapias, praticando a produção dos sons-alvo isolados, associados à vogal [a]. Foi possível perceber que o sujeito conseguiu ver suas falas no monitor de ultrassom em tempo real, enquanto o terapeuta realizava constantes feedbacks verbais, sinalizando o desempenho e permitindo que ele se autoavaliasse cognitivamente sobre os movimentos da neolíngua (Figura 2).

Para se obter os resultados do índice Porcentagem de Consoantes Corretas pré-terapia e pós-terapia, foi realizada a análise quantitativa descritiva e, para se obter os resultados da avaliação perceptivo-auditiva e da autoavaliação, foi realizada a análise qualitativa.

O PCC para os fones tratados [s] e [S], ao longo das duas fases do estudo, apresentou melhora durante a intervenção. No momento da avaliação pré-terapia, a análise dos juízes constatou PCC de grau moderadamente severo $(59,96 \%)$ e, já no momento pós-terapia, observou-se PCC de grau levemente moderado (68,45\%) (Tabela 1$)$.

Em relação aos resultados descritivos quanto ao grau de inteligibilidade de fala observada pelos juízes, em pré-terapia, o indivíduo apresentou inteligibilidade de fala insuficiente $(52,16 \%)$

Quadro 1. Dados da terapia associada ao feedback ultrassonográfico de acordo com cada sessão e seu objetivo

\begin{tabular}{|c|c|c|c|}
\hline \multicolumn{4}{|c|}{ Dados Terapêuticos } \\
\hline Sessões & Objetivos & Instrumentos ou metodologia & Resultados alcançados \\
\hline $1^{\mathrm{a}}$ sessão & Coleta da amostra de fala & $\begin{array}{l}\text { Gravação de dados de imagem } \\
\text { (ultrassom) e áudio por meio da } \\
\text { ferramenta Motu (interface de } \\
\text { áudio). }\end{array}$ & Avaliação pré-intervenção. \\
\hline $2^{\mathrm{a}}$ sessão & Terapia biofeedback & $\begin{array}{l}\text { Percepção da produção dos } \\
\text { fones, movimentos ondulatórios } \\
\text { da neolíngua com o ultrassom. }\end{array}$ & Percepção dos fones [s] e []]. \\
\hline $4^{\mathrm{a}}$ sessão & Terapia biofeedback & $\begin{array}{l}\text { Treino articulatório dos fones [s] } \\
\text { e [J]; sílaba [s]+[a] e [J]+[a] com } \\
\text { ultrassom. }\end{array}$ & $\begin{array}{l}\text { Produção dos fones [s] e [ }[] \\
\text { somado a vogal. }\end{array}$ \\
\hline $5^{\mathrm{a}}$ sessão & Terapia biofeedback & $\begin{array}{l}\text { Treino articulatório do fone [s] } \\
\text { e [J]; sílaba ([s]+[a]) e [J]+[a]; e } \\
\text { palavras com o ultrassom. }\end{array}$ & $\begin{array}{l}\text { Pronúncia sistemática das } \\
\text { palavras com os fones [s] e []]. }\end{array}$ \\
\hline $7^{a}$ sessão & Terapia biofeedback & $\begin{array}{l}\text { Introdução de frases contendo as } \\
\text { palavras trabalhadas em terapia. }\end{array}$ & $\begin{array}{l}\text { Autopercepção quanto a melhora } \\
\text { da inteligibilidade da fala. }\end{array}$ \\
\hline $8^{\mathrm{a}}$ sessão & Terapia biofeedback & $\begin{array}{l}\text { Treino de fala encadeada com } \\
\text { fones fone [s] e [ [] na leitura de } \\
\text { texto com auxílio do ultrassom. }\end{array}$ & $\begin{array}{l}\text { Treino de fala encadeada com o } \\
\text { fone [s] e [J] por meio da leitura de } \\
\text { um texto. }\end{array}$ \\
\hline $9^{a}$ sessão & Terapia biofeedback & $\begin{array}{l}\text { Treino de fala encadeada com os } \\
\text { fones fone [s] e [ }[] \text { na leitura de } \\
\text { texto com auxílio do ultrassom. }\end{array}$ & $\begin{array}{l}\text { Automatização dos fones [s] e [S] } \\
\text { na fala. }\end{array}$ \\
\hline $10^{a}$ sessão & Coleta da amostra de fala & $\begin{array}{l}\text { Gravação de dados de imagem } \\
\text { (ultrassom) e áudio por meio da } \\
\text { ferramenta Motu (interface de } \\
\text { áudio). }\end{array}$ & Avaliação pós-intervenção. \\
\hline
\end{tabular}


e, após a intervenção fonoaudiológica, a inteligibilidade de fala apresentou-se regular (54,16\%) (Tabela 2$)$.

\section{DISCUSSÃO}

Este artigo teve como objetivo verificar os resultados obtidos a partir da intervenção fonoaudiológica com biofeedback da ultrassonografia de língua e caracterizar a produção de fala de um indivíduo com glossectomia total, com o uso de prótese bucomaxilofacial (PBMF). Apesar de o uso do biofeedback por meio da ultrassonografia de língua já ser utilizado em

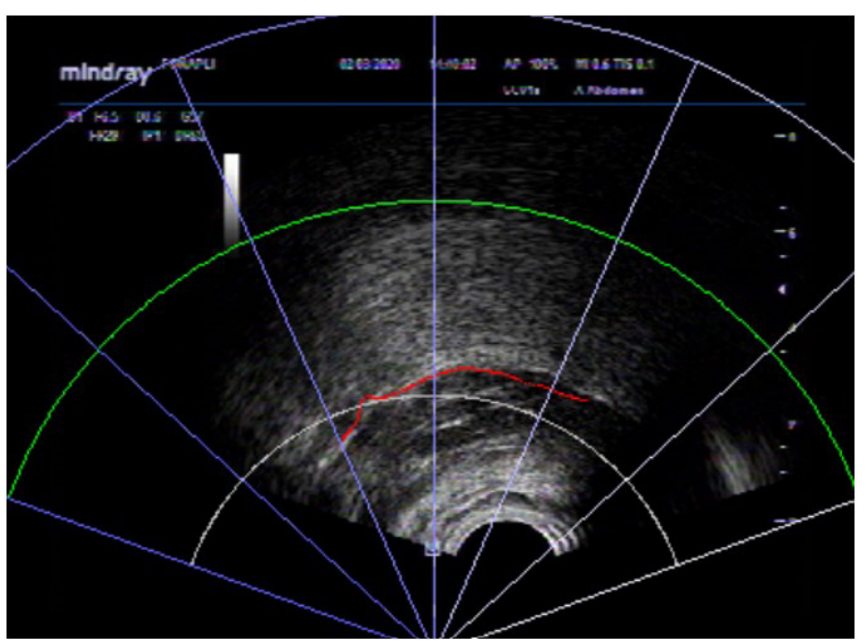

Figura 2. Traçado da superfície da neolíngua em porção anterior e posterior com o uso do biofeedback ultrassonográfico de língua casos de CA de língua, notou-se que ainda são escassos os estudos nessa área, o que revela a importância em investigar as metodologias de intervenção aplicadas, buscando melhor eficácia para o tratamento.

A glossectomia total, principalmente associada à mandibulectomia, pode levar o indivíduo a ter problemas funcionais graves, limitantes, como a fala, a mastigação e a deglutição. Todavia, a eficiência da PBMF melhora cientificamente as habilidades de fala e de deglutição do sujeito, proporcionando a ele melhor qualidade de vida ${ }^{(13,15)}$. Segundo Lauciello et al. ${ }^{(16)}$, indivíduos com movimentos severamente restritos da língua são capazes de melhorar sua inteligibilidade de fala com a modificação palatal proporcionada pela PBMF, devido ao estabelecimento de contatos linguais palatais necessários para se produzir certos sons da fala.

As principais vantagens oferecidas pela PBMF incluem a fácil visualização do defeito local, que permite a detecção de recidivas do CA; a redução do período e dos custos de hospitalização; a capacidade de facilitar o diagnóstico precoce de recidivas e evitar uma segunda operação e o imediato restabelecimento da morfologia facial e das funções orais, como, por exemplo, a função de fala ${ }^{(17)}$.

A alteração na função da fala geralmente é avaliada pela precisão da articulação por meio de PCC-R ${ }^{(13)}$ e pelo GIF. Em relação ao índice de PCC, observou-se que a média do índice do evidenciou melhora de $8,49 \%$ após oito sessões, vide que o aprendizado motor é a retenção de habilidades aprendidas por meio da terapia.

Apesar de o GIF não ser uma medida sensível de precisão da articulação, sua utilização é frequentemente relatada na literatura, quando relacionada ao CA bucal. Logo, focar em um ou dois sons em terapia pode aliviar a dificuldade da tarefa proposta e gerar resultados mais contrastantes ${ }^{(18)}$. Neste estudo, a inteligibilidade dos sons-alvo tratados apresentou mudança

Tabela 1. Medidas descritivas do índice Porcentagem de Consoantes Corretas

\begin{tabular}{cccc}
\hline & PCC & & Dif. \\
\hline Juízes & Pré-terapia & Pós-terapia & 4,03 \\
Avaliador 1 & $58,47 \%$ & $62,50 \%$ & 16,39 \\
Avaliador 2 & $50,83 \%$ & $67,22 \%$ & 5,05 \\
Avaliador 3 & $70,58 \%$ & $75,63 \%$ & 8,49 \\
Média & 59,96 & 68,45 & \\
\hline
\end{tabular}

Legenda: Dif. $=$ diferença; $\mathrm{PCC}=$ Porcentagem de Consoantes Corretas

Tabela 2. Análise de inteligibilidade de fala pré-terapia e pós-terapia

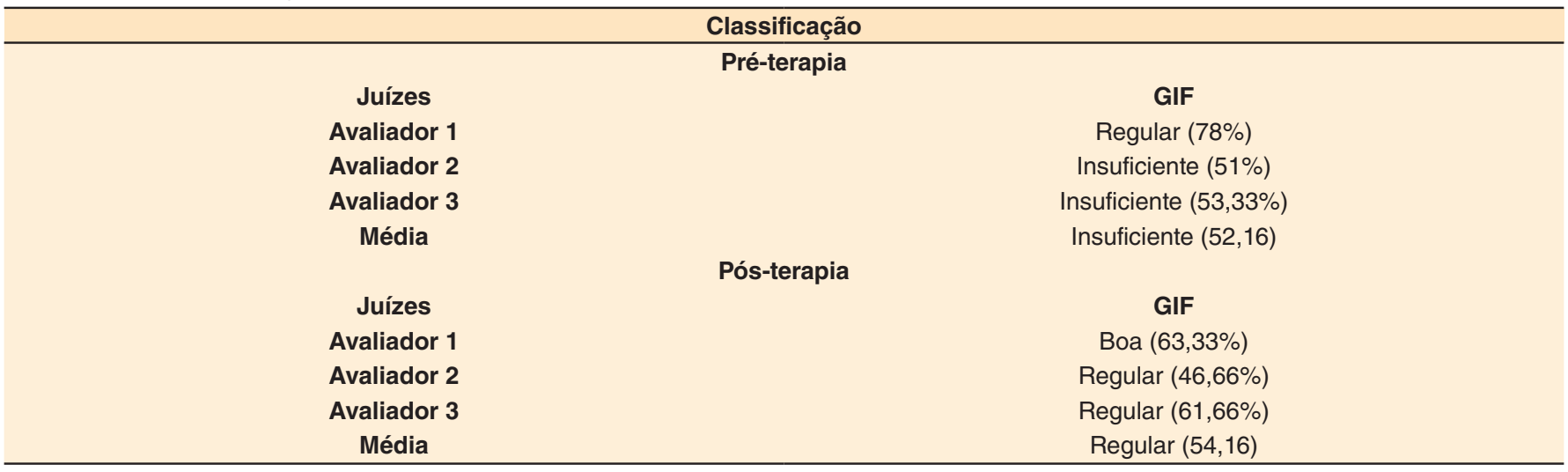


na pronúncia do indivíduo, quando comparada com o prétratamento e o pós-tratamento.

Blyth et al. ${ }^{(8)}$ investigaram os efeitos do biofeedback visual na fala pós-glossectomia em um estudo de caso único de dois pacientes. Os autores acompanharam o progresso da fala dos dois pacientes durante quatro semanas de terapia, relatando resultados melhores para fones tratados e um efeito importante de retenção do fone ao longo do tempo para um paciente. Além disso, estudos demonstram que a reabilitação da fala atua sobre a neuroplasticidade e melhora os resultados da fala após intervenções cirúrgicas de língua. A literatura demonstra que, após a glossectomia, há mudanças adaptativas no córtex cerebral e cerebelo, locais associados ao planejamento dos mecanismos de movimento da língua ${ }^{(19)}$, essenciais para a reabilitação da fala e a deglutição.

No presente estudo, a análise da melhora do sujeito se deu de forma quantitativa e qualitativa, de acordo com os resultados do PCC, da análise perceptivo-auditiva e da própria autoavaliação. Após oito sessões de terapia, foi notória, por artifício das imagens do ultrassom, a diferença do uso da parte posterior da neolíngua, quando comparado com a pré-terapia e a pós-terapia, uma vez que o paciente adaptou a sua produção de fala ao fazer o maior recrutamento dessas estruturas remanescentes.

Não foram encontrados trabalhos que tenham estudado a reabilitação fonoaudiológica com o uso de dispositivo de acrílico semelhante e a avaliação por meio da PCC. Pesquisas sobre a terapia para a reabilitação da fala após ressecção cirúrgica são escassas e não foram encontrados estudos nesse sentido, com o uso de dispositivos individualizados, uma vez que estes são únicos e, com isso, infere-se que sua terapia deva ser individualizada. Os dispositivos intraorais de reabilitação após ressecções devem ser desenvolvidos por meio da estreita colaboração entre o fonoaudiólogo e o cirurgião-dentista especialista. $\mathrm{O}$ atendimento fonoaudiológico, nesses casos, deve visar à manutenção da deglutição de maneira segura e a adaptação da fala e da voz, a partir das estruturas remanescentes e do uso do dispositivo.

Como limitação do estudo, destaca-se a amostra reduzida de produção de fala. Sendo assim, pretende-se realizar avaliações periódicas (ultrassonográficas e perceptivo-auditivas) e incorporar medidas ultrassonográficas quantitativas, a fim de promover a robustez das avaliações e delinear processos terapêuticos mais eficazes para casos semelhantes ao que foi descrito neste trabalho.

\section{COMENTÁRIOS FINAIS}

Após a confecção do dispositivo de acrílico individualizado, foram notadas a melhora da inteligibilidade de fala e a satisfação do sujeito submetido à glossectomia total. A intervenção tradicional somada ao biofeedback visual, por meio do ultrassom, contribuiu para o refinamento e o controle neuromotor da nova estrutura da língua após diversas intervenções cirúrgicas.

\section{AGRADECIMENTOS}

Ao sujeito e aos juízes que participaram da pesquisa.

\section{REFERÊNCIAS}

1. INCA: Instituto Nacional do Câncer. Ministério da Saúde. Câncer de boca - Versão para Profissionais de Saúde [Internet]. 2018 [citado em 2019 Ago 30]. Disponível em: https://www.inca.gov.br

2. AquinoRCA, LimaMLLT, MenezesCRCX, RodriguesM. Alterações fonoaudiológicas e acesso ao fonoaudiólogo nos casos de óbito por câncer de lábio, cavidade oral e orofaringe: um estudo retrospectivo. Rev CEFAC. 2016;18(3):737-45. http://dx.doi.org/10.1590/19820216201618316815 .

3. PauloskiBR, RademakerAW, LogemannJA, McConnelFMS, HeiserMA, CardinaleS, et al. Surgical variables affecting swallowing in patients treated for oral/oropharyngeal cancer.Head Neck. 2004;26(7):625-36. http://dx.doi.org/10.1002/hed.20013. PMid:15229906.

4. ChengSJ, LeeJJ, TingLL, TsengIY, ChangHH, ChenHM, et al. A clinical staging system and treatment guidelines for maxillary osteoradionecrosis in irradiated nasopharyngeal carcinoma patients.Int J Radiat Oncol Biol Phys. 2006;64(1):90-7. http://dx.doi.org/10.1016/j.ijrobp.2005.06.021. PMid: 16213108 .

5. CalheirosAS, AlbuquerqueCL. A vivência da fonoaudiologia na equipe de cuidados paliativos de um Hospital Universitário do Rio de Janeiro.Rev Hosp Univ Pedro Ernesto.2012;11(2):94-8. http://dx.doi. org/10.12957/rhupe.2012.8950.

6. DepprichR, NaujoksC, LindD, OmmerbornM, MeyerU, KüblerNR, et al. Evaluation of the quality of life of patients with maxillofacial defects after prosthodontic therapy with obturator prostheses.Int J Oral Maxillofac Surg. 2011;40(1):71-9. http://dx.doi.org/10.1016/j.ijom.2010.09.019. PMid:20980129.

7. BalasubramaniamMK, ChidambaranathanAS, ShanmugamG, TahR. Rehabilitation of glossectomy cases with tongue prosthesis: a literature review.J Clin Diagn Res. 2016;10(2):ZE01-04. http://dx.doi.org/10.7860/ JCDR/2016/15868.7184. PMid:27042596.

8. BlythKM, MccabeP, MadillC, BallardKJ. Ultrasound visual feedback in articulation therapy following partial glossectomy.J Commun Disord. 2016;61:1-15. http://dx.doi.org/10.1016/j.jcomdis.2016.02.004. PMid:26994583.

9. BressmannT, FlowersH, WongW, IrishJC. Coronal view ultrasound imaging of movement in different segments of the tongue during paced recital: findings from four normal speakers and a speaker with partial glossectomy.Clin Linguist Phon. 2010;24(8):589-601. http:// dx.doi.org/10.3109/02699201003687309.

10. RastadmehrO, BressmannT, SmythR, IrishJC. Increased midsagittal tongue velocity as indication of articulatory compensation in patients with lateral partial glossectomies.Head Neck. 2008;30(6):718-26, discussion 726-7. http://dx.doi.org/10.1002/hed.20772. PMid:18213728.

11. FreedmanSE, MaasE, CaligiuriMP, WulfG, RobinDA. Internal vs. external: oral motor performance as a function of attentional focus.J Speech Lang Hear Res. 2007;50(1):131-6. http://dx.doi.org/10.1044/10924388(2007/011). PMid:17344554.

12. BeumerJ, MarunickMT, EspositoSJ. Maxillofacial rehabilitation: prosthodontic and surgical management of cancer-related, acquired, and congenital defects of the head and neck. Hanover Park: Quintessence Publishing Co., Inc.; 2011.

13. ShribergLD, KwiatkowskiJ. Phonological disorders. I: a diagnostic classification system.J Speech Hear Disord. 1982;47(3):226-41. http:// dx.doi.org/10.1044/jshd.4703.226. PMid:7186559. 
14. HersenM, BarlowDH. Single case experimental designs: strategies for studying behavior change.New York: Pergamon Press; 1982. http:// dx.doi.org/10.1007/978-1-4615-7275-6_7.

15. KharadeP, DholamK, BachherG. Appraisal of function after rehabilitation with tongue prosthesis.J Craniofac Surg. 2018;29(1):e41-4. http:// dx.doi.org/10.1097/SCS.0000000000004008. PMid:29023298.

16. LaucielloFR, VergoT, SchaafNG, ZimmermanR. Prosthodontic and speech rehabilitation after partial and complete glossectomy.J Prosthet Dent. 1980;43(2):204-11. http://dx.doi.org/10.1016/00223913(80)90188-2. PMid:6985967.

17. DepprichR, NaujoksC, LindD, OmmerbornM, MeyerU, KüblerNR, HandschelJ. Evaluation of the quality of life of patients with maxillofacial defects after prosthodontic therapy with obturator prostheses.Int $\mathrm{J}$ Oral Maxillofac Surg. 2011;40(1):71-9. http://dx.doi.org/10.1016/j. ijom.2010.09.019.

18. Girod-RouxM, HueberT, FabreD, GerberS, CanaultM, BedoinN, et al. Rehabilitation of speech disorders following glossectomy, based on ultrasound visual illustration and feedback.Clin Linguist Phon. 2020;34(9):826-43. http://dx.doi.org/10.1080/02699206.2019.1700 310. PMid:31992079.

19. MosierK, LiuWC, BehinB, LeeC, BaredesS. Cortical adaptation following partial glossectomy with primary closure: implications for reconstruction of the oral tongue.Ann Otol Rhinol Laryngol. 2005;114(9):681-7. http:// dx.doi.org/10.1177/000348940511400905. PMid:16240930. 